\title{
Nanoscale mapping by electron energy-loss spectroscopy reveals evolution of organic solar cell contact selectivity
}

Antonio Guerrero ${ }^{1 *}$, Martin Pfannmöller ${ }^{2}$, Alexander Kovalenko, ${ }^{1, \&}$, Teresa S. Ripolles ${ }^{1}$, Hamed Heidari Mezerji ${ }^{2}$, Sara Bals ${ }^{2}$, Louis-Dominique Kaufmann ${ }^{3}$, Juan Bisquert $^{1,4}$, and Germà Garcia-Belmonte ${ }^{1 *}$

1 Photovoltaic and Optoelectronic Devices Group, Departament de Física, Universitat Jaume I, ES-12071 Castelló, Spain

2 EMAT, University of Antwerp, Antwerp, Belgium

3 Genes'Ink, 24 Avenue Gaston Imbert, 13790 Rousset, France

4 Department of Chemistry, Faculty of Science, King Abdulaziz University, Jeddah 21589, Saudi Arabia

\begin{abstract}
Organic photovoltaic (OPV) devices are on the verge of commercialization being long-term stability a key challenge. Morphology evolution during lifetime has been suggested to be one of the main pathways accounting for performance degradation. There is however a lack of certainty on how specifically the morphology evolution relates to individual electrical parameters on operating devices. In this work a case study is created based on a thermodynamically unstable organic active layer which is monitored over a period of one year under non-accelerated degradation conditions. The morphology evolution is revealed by compositional analysis of ultrathin cross-sections using nanoscale imaging in scanning transmission electron microscopy (STEM) coupled with electron energy-loss spectroscopy (EELS). Additionally, devices are electrically monitored in real-time using the non-destructive electrical techniques as capacitancevoltage $(C-V)$ and Impedance Spectroscopy (IS). By comparison of imaging and electrical techniques the relationship between nanoscale morphology and individual electrical parameters of device operation can be conclusively discerned. Is it ultimately observed how the change in the cathode contact properties occurring after the migration of fullerene molecules explains the improvement in the overall device performance.
\end{abstract}

Keywords: Organic photovoltaics, degradation, shelf life, morphology evolution, contact selectivity, low-energy-loss electron spectroscopic imaging. 
\&Permanent address: Brno University of Technology, Faculty of Chemistry, Materials Research Centre, Purkyňova 118, 61200 Brno, Czech Republic

*Corresponding authors: A. Guerrero e-mail: aguerrer@uji.es, G. Garcia-Belmonte, e-mail: garciag@uji.es, tel.: +34 964 387548, fax: +34 964729218

Published in Organic Electronics 16 (2015) 227-233 


\section{Introduction}

Organic photovoltaics (OPV) devices have witnessed an impressive improvement in power conversion efficiencies (PCE) now reaching $10 \%$ for single layer bulk heterojunction (BHJ) solar cells. ${ }^{1}$ However, device stability remains one of the key challenges for their commercialization. There are many factors affecting the device stability with moisture, heat and light regarded as the main stress factors. ${ }^{2}$ Solutionprocessed OPVs are usually based on BHJ films of semiconducting polymers blended with a soluble fullerene derivative that form an interpenetrated bicontinous network. ${ }^{3}$ The organic layer harvests sun light and generates charges via charge transfer from the polymer (donor) to the fullerene (acceptor) that are collected at selective electrodes. ${ }^{3}$ Performance of OPVs is strongly dependent on the donor/acceptor morphology ${ }^{4}$ and variations of the internal arrangement of donor and acceptor molecules is considered to be the main source of efficiency decrease limiting their potential commercialization. ${ }^{5}$

Numerous studies have focused on the importance of the bulk morphology properties of the organic layer. ${ }^{6,7}$ The use of additives ${ }^{8}$, solvent annealing ${ }^{9}$ and thermal treatments ${ }^{10}$ can enhance the crystallinity and would increase the device efficiency. Enhanced crystallinity of the polymer phase have been correlated with high mobility ${ }^{11}$ and improved device performance. ${ }^{12}$ Importantly, intimately mixed donor/acceptor domains are required to provide optimum surface area to enable efficient charge separation. ${ }^{13}$ However, the ability of PCBM molecules to diffuse through the blend film is often regarded as the primary cause of thermally induced degradation of solar cell performance. ${ }^{14}$ The link between morphology modification and performance has usually been discussed in terms of the bulk properties of the active layer, ${ }^{15}$ but very little attention has been paid to the evolving properties of the active layer/outer contact interfaces. For example, molecular orientation of the polymer in intimate contact with the extracting electrode could be limiting the collection ability. ${ }^{16}$ Indeed, morphology of the active layer in BHJ solar cells is frequently the result of a meta-stable configuration and several vertical segregation profiles can be formed. ${ }^{17}$ Thermodynamically stable morphology blends can be obtained by using high transition temperature $\left(T_{\mathrm{g}}\right)$ polymers ${ }^{18}$ or by cross-linking the organic phase ${ }^{19}$. In general terms one of the issues that still require intensive work is the understanding of specific mechanisms behind active layer/outer contact interface chemical and morphological evolution and its relationship to device performance. 
Several morphology characterization techniques are currently available for organic photovoltaic devices and the reader is referred to two recent reviews. ${ }^{20,21}$ For example, advanced techniques such as Grazing Incidence X-ray Diffraction (GXID) ${ }^{22}$ or electron microscopy ${ }^{13,23}$ provide access to the structure and length scales of the donor:acceptor domains. Alternatively, depth profile concentration of donor:acceptor blends can be analyzed by using Dynamic Secondary Ion Mass Spectroscopy (SIMS) ${ }^{24}$, VariableAngle Spectroscopic Ellipsometry (VASE), ${ }^{17}$ Near-Edge X-ray Absorption Fine Structure Spectroscopy (NEXAFS), ${ }^{25}$ etc. On the other hand, advanced electrical techniques are also available which allow understanding individual electrical processes taking place in an operating device. Techniques include Transient Absorption Spectroscopy (TAS) ${ }^{26}$, Transient Photovoltage (TPV $)^{26}$, Capacitance-Voltage $(C-V)^{27}$ or Impedance Spectroscopy (IS) ${ }^{28}$. Interestingly electrical characterization offer the possibility to measure devices using non-destructive methods that enable real-time monitoring.

In this work a case study is created based on a thermodynamically unstable organic layer. The morphology evolution is correlated with the individual electrical processes taking place in the device, and revealed by compositional analysis of ultrathin crosssections using spatially resolved electron energy-loss spectroscopy (EELS) in the analytical scanning transmission electron microscope (STEM). Additionally, devices are electrically monitored in real-time using the non-destructive electrical techniques as $C$ - $V$ and IS. By comparison of imaging and electrical techniques the relationship between morphology and photovoltaic parameter evolution in operating devices is conclusively established. It is ultimately observed how the change in the cathode contact properties occurring after the fullerene molecules migration explains the improvement in the overall device performance. The formation of a PCBM-rich layer contacting the $\mathrm{ZnO}$ electron-selective layer at the cathode causes both the increase in electron selectivity and establishes a good electrical connection between $\mathrm{ZnO}$ and the inner active layer fullerene molecules.

\section{Results}

2.1. Materials and Solar cells fabrication. The method to produce the solar cells is based on standard processing conditions used in the literature ${ }^{29,} 30$ full details can be found as supporting information and a brief description is provided here. Devices were prepared in the inverted configuration ITO/ZnO/P3HT:PCBM/ $\mathrm{MoO}_{3} / \mathrm{Ag}$. Pre-cleaned 
patterned ITO was coated with commercially available $\mathrm{ZnO}$ nanoparticles to provide film thickness of about $50 \mathrm{~nm}$. Substrates were heated at $100{ }^{\circ} \mathrm{C}$ for 5 min followed by a further treatment in the glovebox at $130^{\circ} \mathrm{C}$ for $10 \mathrm{~min}$. Spin coated P3HT:PCBM films $(\sim 100 \mathrm{~nm})$ were thermally treated at $130{ }^{\circ} \mathrm{C}$ for $10 \mathrm{~min}$. Device fabrication was completed by thermal evaporation of $\mathrm{MoO}_{3}(10 \mathrm{~nm})$ and $\mathrm{Ag}(100 \mathrm{~nm})$. Ten independent devices (4 pixels each) were encapsulated with epoxy and a glass slide for electrical characterization. Devices were kept in the glovebox in the dark at all times excluding the time required for electrical characterization ( $<15 \mathrm{~min}$ ). Some un-encapsulated devices were kept in the glovebox for the whole period of the test to characterize the final state of the device using STEM measurements.

2.2. Film and device characterization. Cross-sections of devices were prepared by focused-ion-beam milling using a Helios NanoLab DualBeam (FEI) to a thickness of $\sim 45 \mathrm{~nm}^{31}$ For morphological studies of the cells cast from $\mathrm{CHCl}_{3}$ and to prevent degradation or morphological changes due to storage of the ultrathin cross-sections, STEM investigations were conducted on the same day of FIB preparation. These were done with a Titan QU-Ant-EM microscope (FEI), operated at $120 \mathrm{kV}$ and equipped with a GIF Quantum energy filter (Gatan). STEM dark-field imaging was used for visualization of the integrity of organic and inorganic layers. The pixel size for these images is $0.8 \mathrm{~nm}$, which is sufficient to show the structure the $\mathrm{ZnO}$ layer. Afterwards hyperspectral STEM-SI data sets were acquired across all functional layers at a spatial resolution of $1.3 \mathrm{~nm}$. Electron energy-loss spectra contain contributions from elastic and inelastic scattering up to $90 \mathrm{eV}$ energy-loss, using a dispersion of $0.05 \mathrm{eV} /$ pixel. The applied electron dose for dark-field and hyperspectral images was in sum 300 e/ $\mathrm{A}^{2}$, which was shown to damage the electronic structure of the functional, organic materials but not the bulk plasmon peak differences between P3HT and PCBM. ${ }^{32}$ Data sets were processed and analyzed using Matlab (MathWorks). For this, hyperspectra were normalized to peak intensities and deconvolved to obtain the single-scattering contributions using a script made available by Egerton, ${ }^{31}$ followed by fitting a Gaussian to the plasmon peak. Center positions were mapped out to visualize the morphology of the organic layer. Film thickness was determined either by a VEECO DEKTACK 6M Stylus Profiler or-in case of the cross-sectional thickness-using the energy loss signal. ${ }^{31}$ Current density-voltage characteristics were carried out under illumination with a $1.5 \mathrm{G}$ source $\left(1000 \mathrm{~W} \mathrm{~m}^{-2}\right)$ using an Abet Sun 2000 Solar Simulator. The light intensity 
was adjusted with a calibrated $\mathrm{Si}$ solar cell. Capacitance-voltage and impedance spectroscopy measurements were performed using an Autolab PGSTAT-30 equipped with a frequency analyzer module. Impedance Spectroscopy was carried out under illumination conditions (1sun) using a wide frequency range $(100 \mathrm{~Hz}-1 \mathrm{MHz})$ at several applied DC voltages by applying a small voltage perturbation ( $20 \mathrm{mV} \mathrm{rms})$. Capacitance was recorded at a frequency of $1000 \mathrm{~Hz}$ in the dark at several bias voltages to extract the capacitance-voltage characteristics.

2.3. Shelf-life efficiency. In order to generate a system to study the electrical implications of morphology evolution a polymer with a moderate transition temperature $\left(T_{\mathrm{g}} \sim 120^{\circ} \mathrm{C}\right)^{33} \mathrm{P} 3 \mathrm{HT}$ has been used in combination with a low boiling point solvent $\left(\mathrm{CHCl}_{3}\right)$, followed by thermal treatment. Using these conditions moderate crystalline polymer phases (see Supporting Information for XRD diffraction pattern) are obtained implying that thermodynamically stable morphologies will not be attained. An inverted configuration composed by ITO/ZnO/ $\mathrm{P} 3 \mathrm{HT}: \mathrm{PCBM} / \mathrm{MoO}_{3} / \mathrm{Ag}$ is chosen to minimize any degradation arising from the contacts. Devices are characterized by their current density-voltage $(J-V)$ curves measured over a period of one year using non-accelerated conditions (absence of oxygen, water or light). By using this methodology any change in performance will be due exclusively to a morphology evolution of the active layer blend. As expected for devices processed from chloroform adequate morphology of donor an acceptor is not achieved and initial efficiencies are below $1 \% .^{34}$ The morphology evolution occurring during a period of one year led to a two-fold increase in efficiency. Initial and final photovoltaic parameters are summarized in Table I.

\section{Table I}

Photovoltaic parameters for fresh and aged devices processed under different conditions. Optical and electrical parameters extracted from $J$ - $V$ curves, absorption measurements and capacitance-voltage are included.

\begin{tabular}{ccccccccc}
\hline & & & & $N$ & $V_{F B}$ & Fullerene & Leakage Current \\
cathode & & $($ dark $-1 V)$ \\
Device & $J_{\mathrm{sc}}$ & $V_{\mathrm{oc}}$ & $F F$ & $P C E$ & $\left(\times 10^{16} \mathrm{~cm}^{-3}\right)$ & $(\mathrm{mV})$ & $\begin{array}{c}\text { content } \\
\left(\mathrm{mA} \mathrm{cm}^{-2}\right)\end{array}$ \\
\hline Fresh & 4.4 & 496 & 41 & 0.90 & 4.0 & 610 & 70 & 2.4
\end{tabular}


Figure 1 shows that this system provides a good example of morphology evolving devices. Individual photovoltaic parameters change following two different time trends. Whilst the open circuit voltage $\left(V_{\mathrm{oc}}\right)$ increases dramatically during the first 10-20 hours the short circuit current $\left(J_{\mathrm{sc}}\right)$ increases gradually more than $30 \%$ over the whole period of study. Alternatively, Fill factor (FF) is always stable and low. Overall, the $V_{\mathrm{oc}}$ increases $74 \mathrm{mV}(\sim 10 \%)$ and $J_{\mathrm{sc}}$ increases nearly $3 \mathrm{~mA} / \mathrm{cm}^{2}(45 \%)$. As device performance parameters are the outcome of many different optical and electrical processes, $J-V$ curves cannot help identifying specific mechanisms behind the reported evolution. If deeper knowledge is desired the use of more advance electrical techniques is needed.

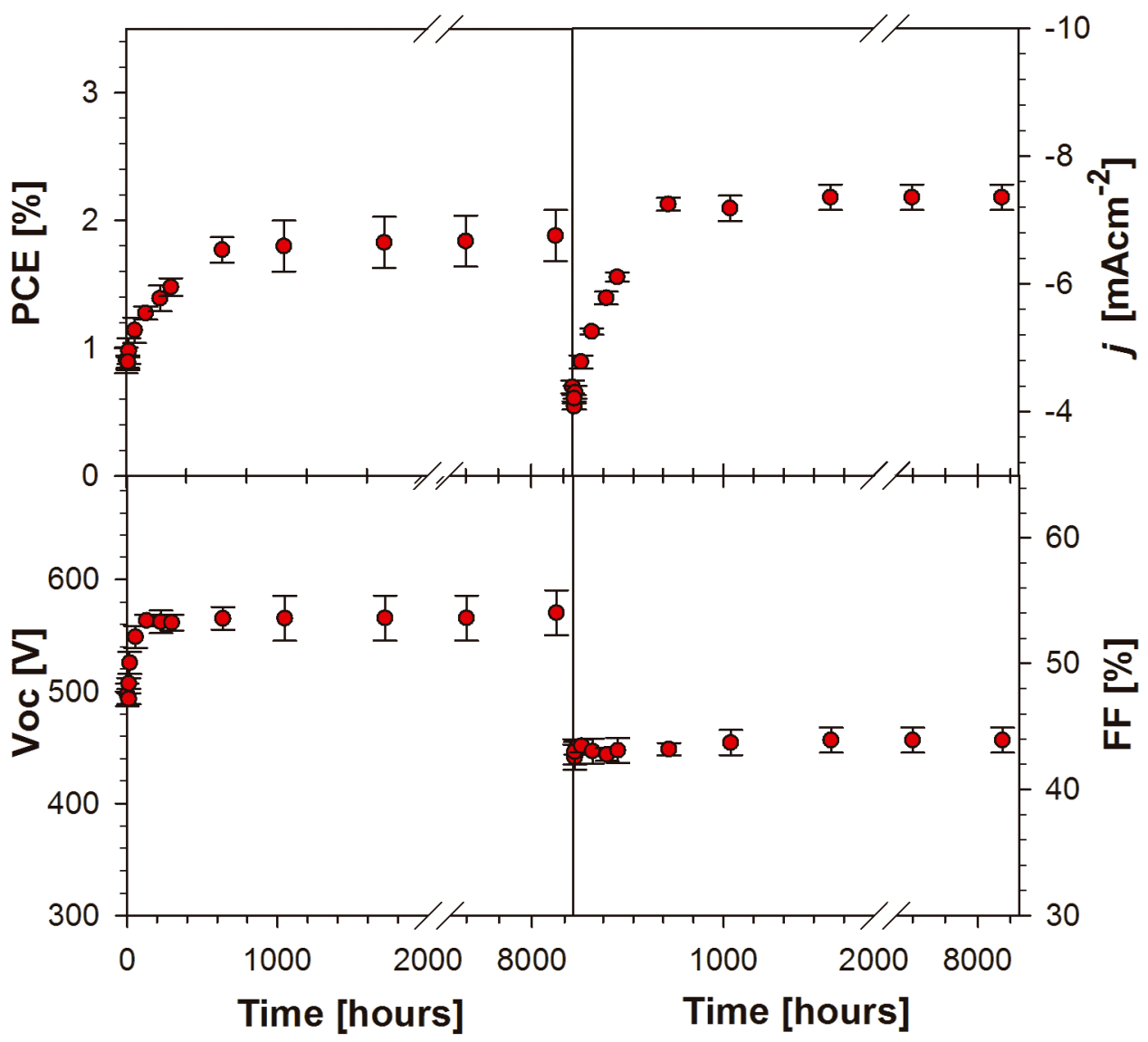

Figure 1: Device performance parameters extracted from $J-V$ curves measured during a period of 1 year for devices fabricated with the architecture $\mathrm{ITO} / \mathrm{ZnO} /$ P3HT:PCBM/ $/ \mathrm{MoO}_{3} / \mathrm{Ag}$ and active layer cast from chloroform $\left(\mathrm{CHCl}_{3}\right)$. 
2.4. Morphology and vertical phase segregation analyzed by electron energy-loss nanoscale mapping. Devices cast from $\mathrm{CHCl}_{3}$ followed by a thermal treatment at $130{ }^{\circ} \mathrm{C}$ pristine and aged have been analyzed using advanced microscopy techniques. We apply STEM in combination with EELS to visualize the morphology of photoactive layers in cross-sections samples prepared using focused ion beam. This combination is called STEM spectral imaging (STEMSI) and offers spatially resolved low energy-loss spectroscopy at the nanoscale. After fitting a Gaussian profile to each spectrum, the determined mean positions can be mapped out. Resulting maps indicate plasmon peak positions, which differ depending on the localized enrichment of a certain material or on the amount of intermixing. Figure $2 \mathrm{a}$ and $2 \mathrm{~b}$ show STEMSI maps of bulk plasmon peak positions, which can typically be found between 20 and $25 \mathrm{eV}$ for carbon based materials. ${ }^{31}$ However, for polymers and fullerenes both center and shape of plasmons differ. ${ }^{13}$ Hence, by acquiring energy loss spectra comprising such low energies, it is possible to obtain reliable, qualitative maps of the morphology at nanometer resolution, applying a relatively small electron dose. These device states correspond to the performance parameters summarized in Table I. As evidenced by such morphological analysis of focused ion beam cross-sections of both fresh and aged samples, fullerene content at the $\mathrm{ZnO}$-covered cathode increases dramatically during the course of the experiment (red color in Figure 2a and 2b). Evolution of morphology in aged samples shows a qualitatively small increase of demixing accompanied by a slight decrease of the intermixed phase, but a highly PCBM-rich layer develops contacting upper $\mathrm{ZnO}$ layer. This observation entails an overall morphological change which produces as a result a vertical segregation of fullerene molecules accumulated at the cathode contact. To verify whether the PCBM accumulation at the cathode contact causes the observed increment in the photovoltaic parameters purely electrical techniques are further used to monitor how fullerene cathode content evolves over time. 

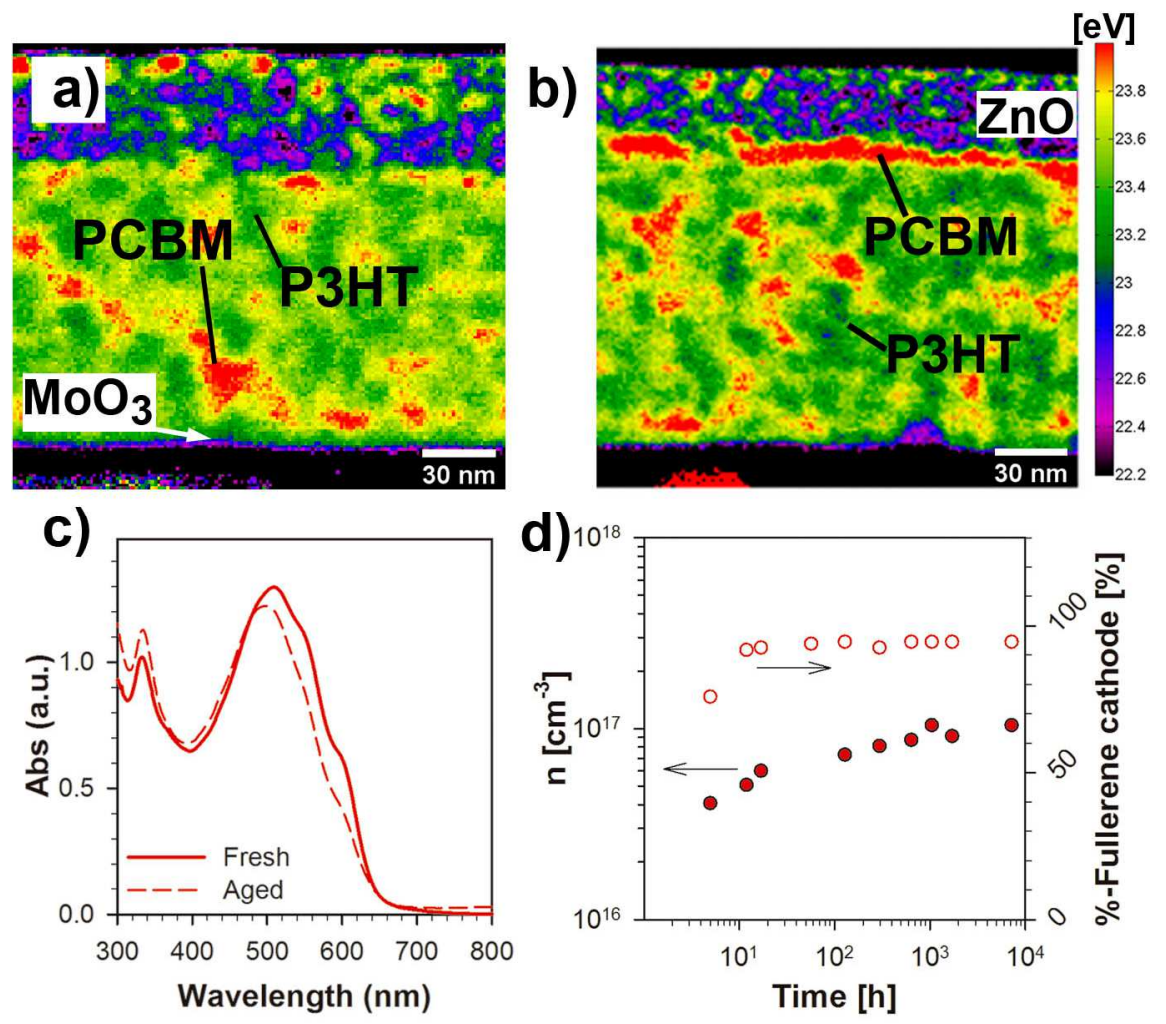

Figure 2: Maps showing plasmon peak positions of ultrathin cross-sections of devices cast from chloroform (a) fresh and (b) aged. Red color denotes PCBM enriched phases (higher plasmon energy) and green color P3HT enriched phases (lower plasmon energy). The maps are created from spatially resolved low energy loss STEMSI data at a resolution of $1.3 \mathrm{~nm}$. Evolution of morphology in aged samples shows that demixing and distribution of domains does not change but that a highly PCBM-rich layer develops at the ZnO-covered contact. (c) Absorption spectra of fresh and aged films cast from $\mathrm{CHCl}_{3}$. (d) Evolution of defect density values (n) with shelf storage and calculated fullerene content at the cathode interface extracted from capacitance-voltage measurements carried out in the dark of devices cast from $\mathrm{CHCl}_{3}$.

2.5 Correlation with electrical measurements. We have recently shown how to extract relevant information about the fullerene content contacting the device cathode by means of capacitance-voltage $(C-V)$ analysis. ${ }^{27,30}$ This technique allows determining the fullerene content at the cathode which is directly related to the contact selectivity. $C-V$ is non-destructive and can be used in complete devices in real time experiments. A small $\mathrm{AC}$ voltage perturbation is applied to a working device at varying DC voltage biases and the differential current output is measured. For OPVs $C-V$ is typically carried out at a certain frequency between $100-1000 \mathrm{~Hz}$ (see Supporting Information for details), and offers information on the energy level equilibration at the organic layer/cathode interface where a dipole layer is generated by reduction of the fullerene molecules present at that interface ${ }^{30}$ Additionally, a confined electrical field at the vicinity of the cathode appears 
producing band bending (depletion zone) in the adjacent active layer.

The fullerene content at the cathode interface is calculated by analysis of the $C-V$ data $^{27}$ as a function of the degradation time, and representative values are shown in Figure 1d. Initially, the fullerene content at the cathode is relatively low $(\sim 70 \%)$ and during the first tens of hours this value increases to nearly full coverage. In addition to the increase in contact selectivity, there is a correlation between fullerene molecules covering the cathode and the leakage current measured under dark conditions (see Table 1 and Figure SI3). ${ }^{27}$ In this case the leakage current decreases over one order of magnitude during the first $20 \mathrm{~h}$. The timescale of this modification correlates with the increase in $V_{\text {oc }}$ as observed in Figure 1.

Along with fullerene content at the cathode, defect density (polymer $p$-doping level) can be extracted from $C-V$ measurements by analyzing Mott-Schottky plots (see Supporting Information for details). It is known that defect density is connected with the crystallinity of the P3HT domains ${ }^{35}$ and with chemical modification due to external dopants. ${ }^{36}$ As devices are not exposed in these experiments to external agents, defect density will be due exclusively to modification in the crystallinity of the P3HT domains. Initial defect levels (Table 1) are higher than those typically observed for relatively high crystalline films cast from ODCB $\left(\sim 5 \times 10^{15} \mathrm{~cm}^{-3}\right){ }^{35}$ This result is in agreement with the low crystallinity of the active layer observed in the XRD diffraction patterns, and absorption measurements (shown as Supporting Information). The defect density increases as a function of time in agreement with the observed reduction in crystallinity as evidenced by the film absorption measurements (Figure 2c). Importantly, the increase in defect density occurs gradually during the whole year (Figure 2d) which is related to the FF as it will be discussed below.

In order to better understand the electrical loses the resistive response of devices are studied by IS under illumination conditions. This technique provides access to series resistance ${ }^{37}$ recombination kinetics ${ }^{38}$ and transport properties. ${ }^{39}$ The working principle of $C$ - $V$ and IS is similar, however, in the later the AC voltage frequency is varied over a wide range $(1 \mathrm{~Hz}-1 \mathrm{MHz})$. Representative spectra are shown as Supporting Information together with the equivalents circuits used to fit the impedance data displayed in Figure 3. The meaning of each of the parameters that can be extracted will be briefly described here. All Nyquist plots show two arcs at high applied voltages, and a contribution at still lower frequencies is observed only for the fresh sample, previously connected to a contact resistance $\left(R_{\mathrm{CO}}\right){ }^{40}$ Values of $30-75 \Omega \mathrm{cm}^{2}$ are observed for $R_{\mathrm{CO}}$ being this a 
significant contribution to the overall series resistance of the device. Interestingly, this resistance is not present in aged devices indicating that in the initial state the fullerene is not able to transfer electrons to the $\mathrm{ZnO}$ increasing as a consequence the contact resistance. In the final state the active layer/ $\mathrm{ZnO}$ interface is enriched with fullerenes and the charge transfer is facilitated reducing the values of $R_{\mathrm{CO}}$ which is no longer observed. As it will be explained below the presence of this $R_{\mathrm{CO}}$ limits the photocurrent extracted in the fresh device.

The impedance features in the high frequency region have previously been correlated with resistive processes caused by carrier movement $\left(R_{\mathrm{tr}}\right)$, also contributing to the series resistance of the device. ${ }^{37}$ This is normally observed in non optimized devices when morphology issues limit efficient transport of carriers. ${ }^{28,} 39$ For fresh devices $R_{\mathrm{tr}}$ is relatively small 2-15 $\Omega \mathrm{cm}^{2}$ indicating that the low FF observed is the consequence of the high $R_{\mathrm{CO}}$. On the other hand, aged devices show values of about $20-100 \Omega \mathrm{cm}^{2}$. This notable increase in $R_{\mathrm{tr}}$ is correlated with the increase in defect density that impedes the efficient transport of carriers. The low frequency impedance response corresponds to the recombination resistance $\left(R_{\text {rec }}\right)$ present in all photovoltaic devices, which represents the opposition of the system to free carrier recombination. ${ }^{38}$ High $R_{\text {rec }}$ are desired as this is a sign of low recombination fluxes. ${ }^{41}$ At low applied voltages the extracted $R_{\text {rec }}$ shows similar values for fresh and aged devices indicating that the reduction of $R_{\mathrm{CO}}$ for the aged device is totally compensated by the increase in $R_{\text {tr }}$. However, for the fresh device and above $0.25 \mathrm{~V}$ the system is not able to increase the photogenerated carrier density (related to the values attained by the chemical capacitance $C_{\mu}$ ) and recombination takes place more readily. 


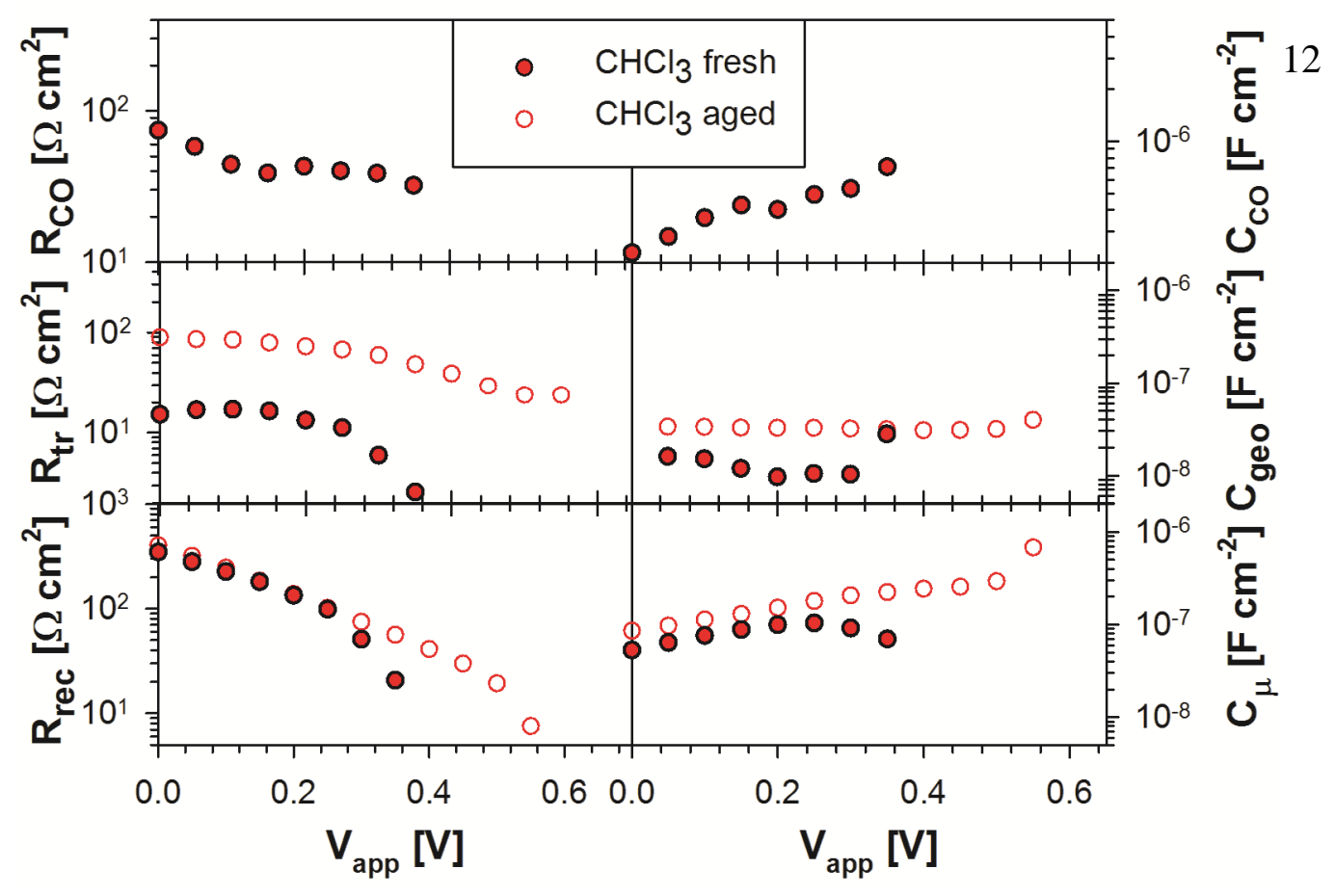

Figure 3: Fitting results extracted from impedance spectroscopy data for representative devices measured in the initial state (fresh) and at the end of the lifetime (aged). The equivalent circuits used are shown as supporting information.

\section{Discussion}

As discussed previously, shelf life evolution of photovoltaic parameters in Figure 1 exhibit three different trends. $V_{\text {oc }}$ shows a subtle increment within the first $100 \mathrm{~h}$ and then remains stable. $J_{\text {sc }}$ evolves much slowly and reaches its maximum after $600 \mathrm{~h}$. On the contrary FF remains rather constant throughout the experiment. Long term stability does not change any more as observed in Figure 1a. It is known that the origin of the $V_{\mathrm{oc}}$ is related to energetic ${ }^{42}$ and kinetic mechanisms. ${ }^{43}$ Indeed, the difference between the donor HOMO and the acceptor LUMO determines $V_{\mathrm{oc}}$ as a first approximation. ${ }^{44}$ In addition, gap defect states are known to modulate the Fermi level position that ultimately establishes the output $V_{\text {oc. }}{ }^{35}$ Increased defect density in the aged device should in theory decrease the $V_{\text {oc }}$ as these defects should reduce Fermi level shift upon illumination. However, in this work $V_{\text {oc }}$ increases and results from STEM, capacitancevoltage and impedance spectroscopy provide a clear answer on the nature of such an improvement. This increase follows the same time scale as the increase in the contact selectivity indicating that originally the device was seriously limited by an inefficient connection between the cathode and the active layer fullerene molecules. Moreover this poor electrical connection is evidenced by the large contact resistance extracted from IS of fresh devices. After morphology evolution caused by the presence of meta-stable phases, the cathode contact gains fullerene molecules, becoming more electron selective. 
Consequently the resistance associated to the contact electron hindrance disappears allowing for the $V_{\mathrm{oc}}$ increase. The presence of a sufficiently high fullerene covering of the cathode interface as a necessary condition for efficient device operation was demonstrated in previous work. ${ }^{27}$

Balance among different resistive mechanisms lies behind the stable trend exhibited by FF. Three different contributions are expected to influence FF: $(i)$ a reduction in the overall series resistance mainly caused by decrease in $R_{\mathrm{CO}}$, (ii) increase in transport resistance and (iii) the observation of similar recombination kinetics. The overall series resistance of OPV devices is dominated by the resistance associated to the contacts. ${ }^{45}$ Here very high contact resistances are found for fresh devices of up to $75 \Omega \mathrm{cm}^{2}$ at $0 \mathrm{~V}$ (Figure 3) that in addition is clearly limiting the extracted charge. Then the series resistance decreases during the lifetime as $R_{\mathrm{CO}}$ is not present for aged devices. On the other hand, $R_{\mathrm{tr}}$ is also a source of resistive loses and in all cases this resistive contribution is observed for devices casted from $\mathrm{CHCl}_{3}{ }^{46}$ However, the magnitude of this parameter is relatively low for fresh devices $\left(>20 \Omega \mathrm{cm}^{-2}\right)$ and increases with the presence of more electrically active defects $\left(20-85 \Omega \mathrm{cm}^{-2}\right)$. It is noted that the increment in $R_{\mathrm{tr}}$ roughly compensates the enhanced cathode connection $\left(R_{\mathrm{CO}}\right.$ disappearance). Finally, values of $R_{\text {rec }}$ as a function of the applied bias are very similar. Overall, the improvement in the series resistance appears to be dominant over the increase in the resistance related to transport as the FF slightly increases during the test.

Increment in $J_{\mathrm{sc}}$ during the first $600 \mathrm{~h}$ should be related to a combination of several factors. The maximum current calculated by integration of the absorption spectra (Table 1) provides values of $12 \mathrm{~mA} \mathrm{~cm}$. This is the current that could be obtained if no electrical losses would be taking place. However, only 4-7 $\mathrm{mA} \mathrm{cm}^{-2}$ are extracted at short circuit. Therefore, almost half of photons are lost due to inefficient physical processes occurring during device operation. As no significant difference in absorption degree between fresh and aged films occurs (see Figure 2c) the increase in $J_{\mathrm{sc}}$ must arise from the partial suppression of inner loss mechanisms. In devices limited by high series resistance $J_{\text {sc }}$ may be seriously affected. ${ }^{46}$ An important decrease in $R_{\mathrm{CO}}$ takes place during the lifetime, being no longer observed after the device test, and this enables a more efficient extraction of carriers to the electrodes and improves collecting efficiency. However, contact selectivity increment (inferred from the fullerene covering in Figure $2 \mathrm{~d})$ occurs subtly after device construction $(<100 \mathrm{~h})$. Alternatively, the transport resistance $R_{\text {tr }}$ is observed to increase during the experiment in good agreement with the 
augment of the defect density in Figure 2d. An additional factor which contributes to the collected current is the increment in photogenerated carrier density. Using analytical transmission electron microscopy it was previously shown that the extracted photocurrent largely depends on the nanoscale morphology features. ${ }^{13}$ Here, we observe a qualitatively small increase of demixing accompanied by a slight decrease of the intermixed phase (Figure 2a and 2b). Importantly, this modification of the morphology in the bulk of the active layer is expected to take place gradually over the whole study, and might assist photogeneration. This final observation is in agreement with the reduction in polymer crystallinity as inferred from absorption data in Figure 2c.

\section{Conclusion}

In summary, a case study that provides morphology evolving organic layers is generated and initial and final states have been analyzed by advanced microscopy techniques. Compositional analysis of ultrathin cross-sections using spatially resolved electron energy-loss spectra in the analytical STEM reveals that active layer morphology evolves forming a PCBM-rich layer at the organic layer/ZnO interface. Evolution in morphology yields a change in the vertical segregation profile that lead to an increase in the contact selectivity accompanied by a good electrical connection between the cathode and active layer fullerene molecules. Devices are further electrically characterized by using $C$ - $V$ and IS techniques to confirm the increase in fullerene covering at the cathode interface. We highlight how the change in the cathode contact properties occurring after the fullerene molecules migration explains the improvement in the overall device performance. 
Associated content.

Supporting Information. Detailed XDR, absorption spectra, Mott-Schottky analysis, and Impedance Spectroscopy experiments are shown.

\section{Author Information.}

\&Permanent address: Brno University of Technology, Faculty of Chemistry, Materials Research Centre, Purkyňova 118, 61200 Brno, Czech Republic

*Corresponding authors: A. Guerrero e-mail: aguerrer@uji.es, G. Garcia-Belmonte, e-mail: garciag@uji.es, tel.: +34 964 387548, fax: +34 964729218

\section{Acknowledgement.}

This work was partially supported by FP7 European collaborative project SUNFLOWER (FP7-ICT-2011-7-contract num. 287594), and Generalitat Valenciana (project ISIC/2012/008 Institute of Nanotechnologies for Clean Energies). S.B. acknowledges financial support from European Research Council (ERC Starting Grant \#335078-COLOURATOMS). A.K. acknowledges Brno University of Technology for financial support (CZ.1.07/2.3.00/30.0039). M.P. gratefully acknowledges the SIM NanoForce program for their financial support. 


\section{References}

1. He, Z.; Zhong, C.; Su, S.; Xu, M.; Wu, H.; Cao, Y. Nature Photonics 2012, 6, (9), 591-595.

2. Jørgensen, M.; Norrman, K.; Gevorgyan, S. A.; Tromholt, T.; Andreasen, B.; Krebs, F. C. Advanced Materials (Weinheim, Germany) 2012, 24, (5), 580-612.

3. Yu, G.; Gao, J.; Hummelen, J. C.; Wudl, F.; Heeger, A. J. Science 1995, 270, (5243), 1789-1791.

4. Yang, X.; Loos, J.; Veenstra, S. C.; Verhees, W. J. H.; Wienk, M. M.; Kroon, J. M.; Michels, M. A. J.; Janssen, R. A. J. Nano Letters 2005, 5, (4), 579-583.

5. Li, Z.; Wong, H. C.; Huang, Z.; Zhong, H.; Tan, C. H.; Tsoi, W. C.; Kim, J. S.; Durrant, J. R.; Cabral, J. T. Nat Commun 2013, 4, 2227.

6. Günes, S.; Neugebauer, H.; Sariciftci, N. S. Chemical Reviews (Washington, DC, United States) 2007, 107, (4), 1324-1338.

7. Brady, M. A.; Su, G. M.; Chabinyc, M. L. Soft Matter 2011, 7, 11065.

8. Dang, M. T.; Wuest, J. D. Chemical Society Reviews 2013, 42, (23), 9105-9126.

9. Li, G.; Shrotriya, V.; Huang, J.; Yao, Y.; Moriarty, T.; Emery, K.; Yang, Y. Nature Materials 2005, 4, (11), 864-868.

10. Ma, W.; Yang, C.; Gong, X.; Lee, K.; Heeger, A. J. Advanced Functional Materials 2005, 15, (10), 1617-1622.

11. Sirringhaus, H.; Tessler, N.; Friend, R. H. Science 1998, 280, (5370), 1741-1744.

12. Chen, Y.; Wan, X.; Long, G. Accounts of Chemical Research 2013, 46, (11), 2645-2655.

13. Pfannmöller, M.; Flügge, H.; Benner, G.; Wacker, I.; Sommer, C.; Hanselmann, M.; Schmale, S.; Schmidt, H.; Hamprecht, F. A.; Rabe, T.; Kowalsky, W.; Schröder, R. R. Nano Letters 2011, 11, 3099-3107.

14. Ebadian, S.; Gholamkhass, B.; Shambayati, S.; Holdcroft, S.; Servati, P. Solar Energy Materials and Solar Cells 2010, 94, (12), 2258-2264.

15. Jamieson, F. C.; Domingo, E. B.; McCarthy-Ward, T.; Heeney, M.; Stingelin, N.; Durrant, J. R. Chemical Science 2012, 3, (2), 485-492.

16. Collins, B. A.; Cochran, J. E.; Yan, H.; Gann, E.; Hub, C.; Fink, R.; Wang, C.; Schuettfort, T.; McNeill, C. R.; Chabinyc, M. L.; Ade, H. Nature Materials 2012, 11, (6), 536-543.

17. Campoy-Quiles, M.; Ferenczi, T.; Agostinelli, T.; Etchegoin, P. G.; Kim, Y.; Anthopoulos, T. D.; Stavrinou, P. N.; Bradley, D. D. C.; Nelson, J. Nature Materials 2008, 7, (2), 158-164.

18. Sachs-Quintana, I. T.; Heumüller, T.; Mateker, W. R.; Orozco, D. E.; Cheacharoen, R.; Sweetnam, S.; Brabec, C. J.; McGehee, M. D. Advanced Functional Materials 2014, 24, (25), 3978-3985. 
19. Li, Z.; Wong, H. C.; Huang, Z.; Zhong, H.; Tan, C. H.; Tsoi, W. C.; Kim, J. S.; Durrant, J. R.; Cabral, J. T. Nat Commun 2013, 4.

20. Chen, W.; Nikiforov, M. P.; Darling, S. B. Energy and Environmental Science 2012, 5, (8), 8045-8074.

21. Pfannmöller, M.; Kowalsky, W.; Schröder, R. R. Energy \& Environmental Science 2013, 6, 2871-2891.

22. Rivnay, J.; Mannsfeld, S. C. B.; Miller, C. E.; Salleo, A.; Toney, M. F. Chemical Reviews (Washington, DC, United States) 2012, 112, 5488-5519.

23. Bavel, S. S. v.; Sourty, E.; With, G. d.; Loos, J. Nano Letters 2009, 9, 507-513.

24. Björström, C. M.; Nilsson, S.; Bernasik, A.; Budkowski, A.; Andersson, M.; Magnusson, K. O.; Moons, E. Applied Surface Science 2007, 253, (8), 3906-3912.

25. Zhu, S.; Liu, Y.; Rafailovich, M. H.; Sokolov, J.; Gersappe, D.; Winesett, D. A.; Ade, H. Nature 1999, 400, (6739), 49-51.

26. Grancini, G.; Polli, D.; Fazzi, D.; Cabanillas-Gonzalez, J.; Cerullo, G.; Lanzani, G. The Journal of Physical Chemistry Letters 2011, 2, (9), 1099-1105.

27. Guerrero, A.; Dörling, B.; Ripolles-Sanchis, T.; Aghamohammadi, M.; Barrena, E.; Campoy-Quiles, M.; Garcia-Belmonte, G. ACS Nano 2013, 7, (5), 4637-4646.

28. Guerrero, A.; Montcada, N. F.; Ajuria, J.; Etxebarria, I.; Pacios, R.; GarciaBelmonte, G.; Palomares, E. Journal of Materials Chemistry A 2013, 1, (39), 12345-12354.

29. Ajuria, J.; Etxebarria, I.; Cambarau, W.; Munecas, U.; Tena-Zaera, R.; Jimeno, J. C.; Pacios, R. Energy \& Environmental Science 2011, 4, (2), 453-458.

30. Guerrero, A.; Marchesi, L. F.; Boix, P. P.; Bisquert, J.; Garcia-Belmonte, G. The Journal of Physical Chemistry Letters 2012, 3, (10), 1386-1392.

31. Egerton, R. F., Electron Energy-Loss Spectroscopy in the Electron Microscope. Springer: New York, 2011.

32. Pfannmöller, M.; Flügge, H.; Benner, G.; Wacker, I.; Kowalsky, W.; Schröder, R. R. Synthetic Metals 2012, 161, (23-24), 2526-2533.

33. Lungenschmied, C.; Bauer, S.; Schwödiauer, R.; Rodman, S.; Fournier, D.; Dennler, G.; Brabec, C. J. Journal of Applied Physics 2011, 109, (4), 044503044503-5.

34. Miller, S.; Fanchini, G.; Lin, Y.-Y.; Li, C.; Chen, C.-W.; Su, W.-F.; Chhowalla, M. Journal of Materials Chemistry 2008, 18, (3), 306-312.

35. Ripolles, T. S.; Guerrero, A.; Garcia-Belmonte, G. Applied Physics Letters 2013, 103, (24), 243306.

36. Guerrero, A.; Boix, P. P.; Marchesi, L. F.; Ripolles-Sanchis, T.; Pereira, E. C.; Garcia-Belmonte, G. Solar Energy Materials and Solar Cells 2012, 100, (0), 185191. 
37. Guerrero, A.; Ripolles-Sanchis, T.; Boix, P. P.; Garcia-Belmonte, G. Organic Electronics 2012, 13, (11), 2326-2332.

38. Boix, P. P.; Guerrero, A.; Marchesi, L. F.; Garcia-Belmonte, G.; Bisquert, J. Advanced Energy Materials 2011, 1, (6), 1073-1078.

39. Guerrero, A.; Loser, S.; Garcia-Belmonte, G.; Bruns, C. J.; Smith, J.; Miyauchi, H.; Stupp, S. I.; Bisquert, J.; Marks, T. J. Physical Chemistry Chemical Physics 2013, 15, (39), 16456-16462.

40. Guerrero, A.; Chambon, S.; Hirsch, L.; Garcia-Belmonte, G. Advanced Functional Materials 2014, DOI: 10.1002/adfm.201401233.

41. Ripolles-Sanchis, T.; Raga, S. R.; Guerrero, A.; Welker, M.; Turbiez, M.; Bisquert, J.; Garcia-Belmonte, G. The Journal of Physical Chemistry C 2013, 117, (17), 8719-8726.

42. Brabec, C. J.; Cravino, A.; Meissner, D.; Sariciftci, N. S.; Fromherz, T.; Rispens, M. T.; Sanchez, L.; Hummelen, J. C. Advanced Functional Materials 2001, 11, (5), 374-380.

43. Bisquert, J.; Garcia-Belmonte, G. The Journal of Physical Chemistry Letters 2011, 2, 1950-1964.

44. Widmer, J.; Tietze, M.; Leo, K.; Riede, M. Advanced Functional Materials 2013, 23, 5814-5821.

45. Ripolles-Sanchis, T.; Guerrero, A.; Bisquert, J.; Garcia-Belmonte, G. The Journal of Physical Chemistry C 2012, 116, (32), 16925-16933.

46. Steim, R.; Kogler, F. R.; Brabec, C. J. Journal of Materials Chemistry 2010, 20, (13), 2499-2512. 


\section{Supporting Information}

\section{Nanoscale mapping by electron energy-loss spectroscopy reveals evolution of organic solar cell contact selectivity}

Antonio Guerrero ${ }^{1}$, Martin Pfannmöller $^{2}$, Alexander Kovalenko ${ }^{1, \&}$, Teresa S. Ripolles $^{1}$, Hamed Heidari Mezerji ${ }^{2}$, Sara Bals ${ }^{2}$, Louis-Dominique Kaufmann ${ }^{3}$, Juan Bisquert $^{1,4}$, and Germà Garcia-Belmonte ${ }^{1 *}$

1 Photovoltaic and Optoelectronic Devices Group, Departament de Física, Universitat Jaume I, ES-12071 Castelló, Spain

2 EMAT, University of Antwerp, Antwerp, Belgium

3 Genes'Ink, 24 Avenue Gaston Imbert, 13790 Rousset, France

4 Department of Chemistry, Faculty of Science, King Abdulaziz University, Jeddah 21589, Saudi Arabia 


\section{Crystallinity characterization of films by XRD vs absorption}

Crystallinity of the P3HT:PCBM organic phases can be discussed in terms of the XRD diffraction pattern and their film absoption characteristics. Here we compare de crystallinity of devices cast from $\mathrm{CHCl}_{3}$ with those cast from o-dichlorobencene (ODCB) as the later is known to provide relatively high crystalline phases. Then, we analyze the bulk properties of the blend in the absence of the top contact. Crystallinity of the polymer phase is important for the performance of the blend as high crystalline phases enhance the transport of carriers and can improve the device efficiency. ${ }^{[30]}$ The crystallinity of the blend can be assessed by analysis of the XRD pattern from fresh films casted over glass/ITO/ZnO (Figure SI1). The diffraction peak at about $2 \theta \sim 5.5^{\circ}$ corresponds to the $a$-axis orientation related to the packing of the alkyl chains of the P3HT crystallites. Interestingly, the intensity of this peak is significantly higher for samples casted from ODCB compared to those casted from $\mathrm{CHCl}_{3}$ indicating the ODCB sample is more crystalline. Note that in both cases the samples received a thermal treatment and relatively high crystalline samples are expected. From the width and position of the diffraction peak an estimation of the P3HT nanodomain size is calculated for $\mathrm{ODCB}$ and $\mathrm{CHCl}_{3}$ to provide values of 20 and $10 \mathrm{~nm}$, respectively. ${ }^{[28]}$ Similarly, crystallinity can be discussed in terms of variation in absorption features, particularly the position of the maximum absorption at $\sim 500 \mathrm{~nm}$ and its relative height with the vibronic

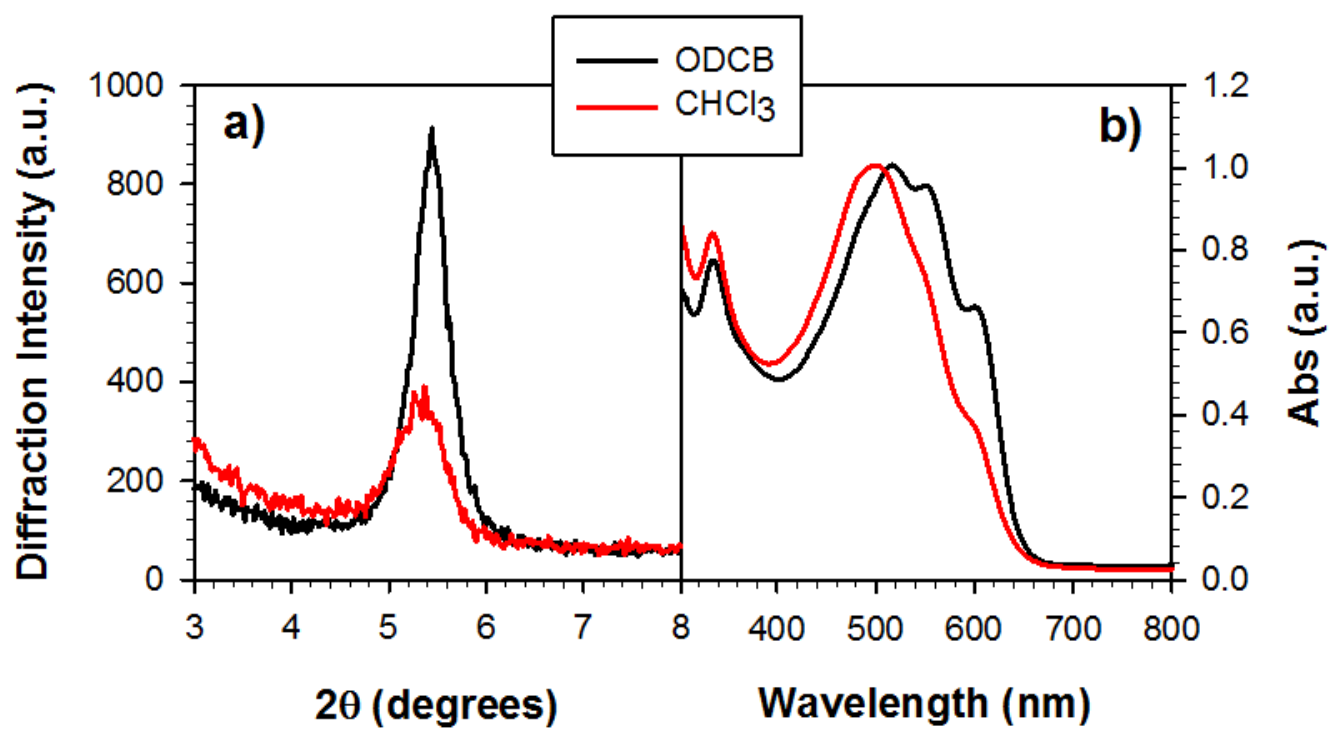

Figure SI1: a) XDR diffraction pattern and b) absorption spectra of films casted over ITO/ZnO substrates using either from o-dichlorobenzene (ODCB) or chloroform 
$\left(\mathrm{CHCl}_{3}\right)$ as solvents.

shoulder at $600 \mathrm{~nm}$. Figure 4b clearly shows that the sample casted from ODCB shows red shifted absorption of the peak at about $500 \mathrm{~nm}$ and higher intensity for the shoulder at about $600 \mathrm{~nm}$. Both features have been previously correlated with increased crystallinity of the P3HT domains. Unfortunately, both XRD and absorption measurements can only be applied for fresh devices in the absence of the top contact or after termination of the life test as their use in aged devices can only be carried out through destructive methods.

Figure SI2 exhibits representative the Mott-Schottky plots and their evolution during solar cells testing life. Mott-Schottky analysis measures the contact capacitance as a function of the applied voltage at reverse and low-forward bias. As the voltage progresses in the positive direction the depletion layer formed at the cathode contact is narrowed. This voltage-induced effect yields an increment in the contact capacitance in agreement with the known equation $C^{-2}=2\left(V_{b i}-V\right) / q \varepsilon N$. The previous relation yields a straightline as a function of the bias from which the defect density $N$ can be extracted. As the slope is inversely proportional to $N$ it is inferred that defect density increases with time.

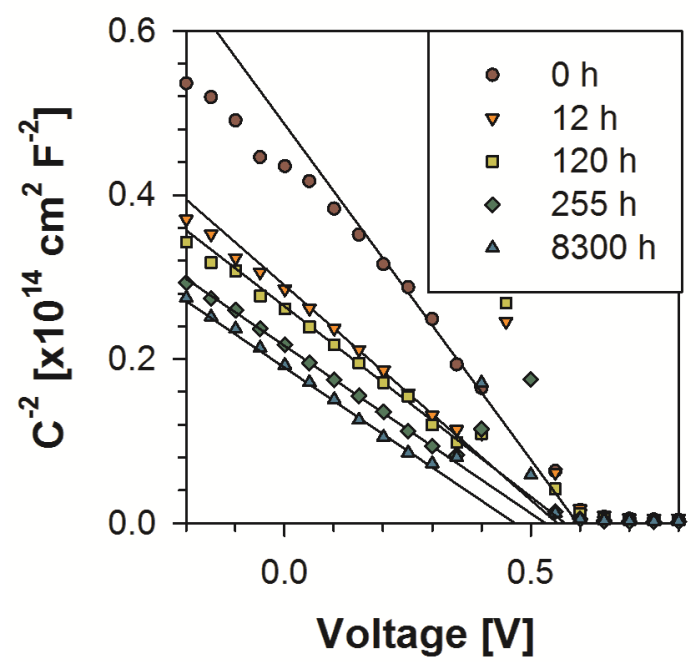

Figure SI2: Evolution of Mott-Schottky response that explains the improvement in contact selectivity during the device test. 


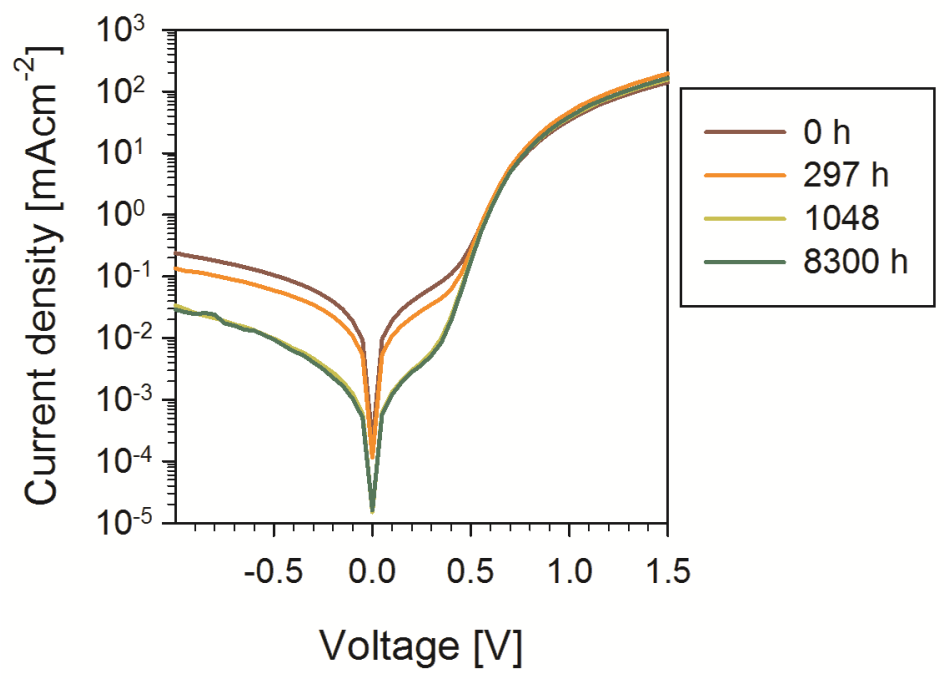

Figure SI3: Evolution of diode curves in dark conditions.

Previous plot (Fig. SI3) evidences a decrease in the diode leakage current with tests time. This evidence informs on the enhancement in the selectivity of the contacts during the device test. 

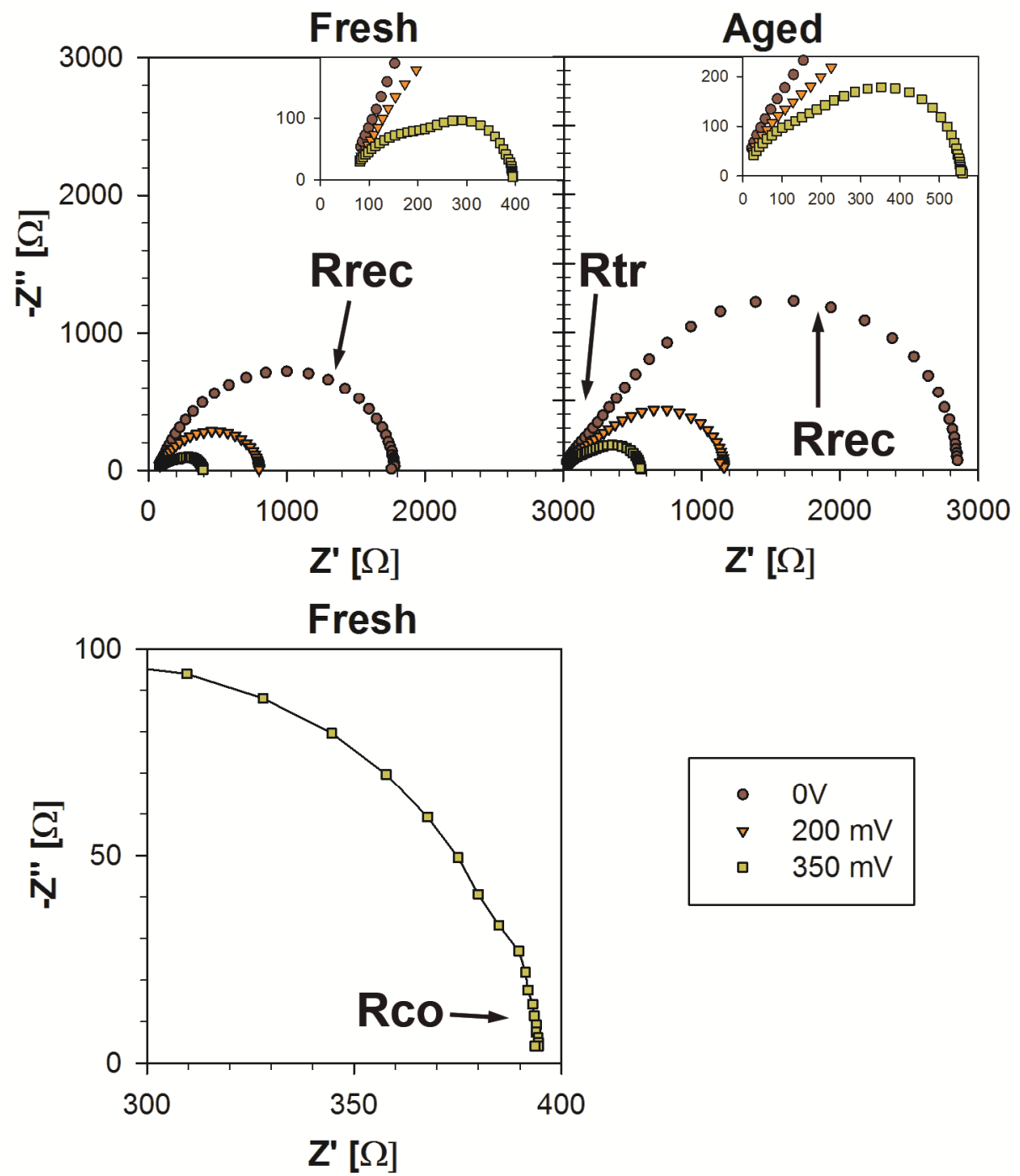

Figure SI4: Nyquist plots of representative fresh and aged devices.

Fig. SI4 shows impedance plots of fresh and aged solar cells. It is clearly observed that in both cases an equivalent circuit comprising two subcircuits (semiarcs) is observed (see Fig. SI5a). In the case of fresh samples a additional feature is observed at lowfrequencies (marked as Rco contribution) that is modeled by means of an additional arc as proposed in Fig. SI5b. 
a)

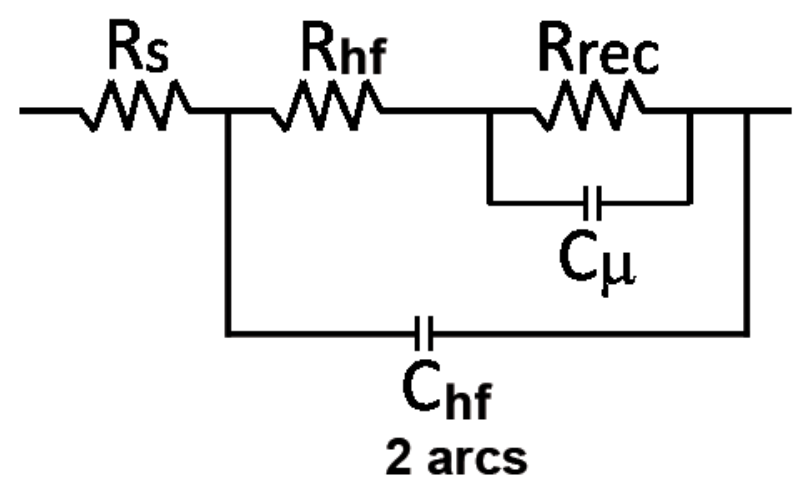

b)

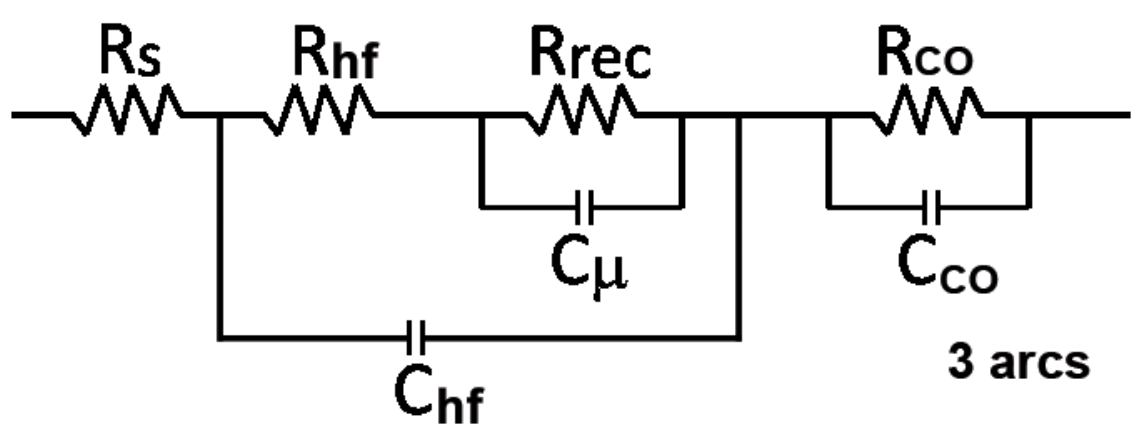

Figure SI5: Equivalent circuits used for spectra responding to two (aged) and three $\operatorname{arcs}($ fresh). 